\title{
YOUNG STARS AT THE EDGE: STELLAR CLUSTERING IN THE OUTER REGIONS OF THE M33 DISK ${ }^{1}$
}

\author{
T. J. Davidge \\ Herzberg Institute of Astrophysics, \\ National Research Council of Canada, 5071 West Saanich Road, \\ Victoria, BC Canada V9E 2E7 \\ email: tim.davidge@nrc.ca \\ T. H. Puzia \\ Department of Astronomy and Astrophysics, \\ Pontifica Universidad Catolica, Santiago, Chile 7820436 \\ email: tpuzia@gmail.com \\ A. W. McConnachie \\ Herzberg Institute of Astrophysics, \\ National Research Council of Canada, 5071 West Saanich Road, \\ Victoria, BC Canada V9E 2EY \\ email: alan.mcconachie@nrc.ca
}

\begin{abstract}
We investigate the distribution of bright main sequence stars near the northern edge of the M33 disk. Clustering on sub-kpc scales is seen among stars with ages $\sim 10 \mathrm{Myr}$, and two large star-forming complexes are identified. Similar large-scale grouping is not evident among stars with ages 100 Myr. These stars are also distributed over a much larger area than those with younger ages, and it is argued that random stellar motions alone, as opposed to orderly motions of the type spurred by large scale secular effects, can re-distribute stars out to distances of at least $2 \mathrm{kpc}$ (i.e. one disk scale length) from their birth places on $100 \mathrm{Myr}$ timescales. Such random motions may thus play a significant role in
\end{abstract}

\footnotetext{
${ }^{1}$ Based on observations obtained with MegaPrime/MegaCam, a joint project of CFHT and CEA/DAPNIA, at the Canada-France-Hawaii Telescope (CFHT) which is operated by the National Research Council (NRC) of Canada, the Institut National des Science de l'Univers of the Centre National de la Recherche Scientifique (CNRS) of France, and the University of Hawaii.
} 
populating the outer regions of the M33 disk. Finally, it is suggested that - to the extent that the ambient properties of the outer disk mirror those in the main body of the disk - stars in this part of M33 may have formed in star clusters with masses $50-250 \mathrm{M}_{\odot}$, which is substantially lower than the peak of the solar neighborhood initial cluster mass function.

Subject headings: galaxies: evolution - galaxies: spiral — galaxies: individual(M33)

\section{INTRODUCTION}

The stellar disks of spiral galaxies can extend to many scale lengths (e.g. Davidge 2006; Pohlen \& Trujillo 2006), and the stars that populate the peripheral regions of disks likely have a range of origins. Some of the stars at large radii probably formed in situ. Ultraviolet light concentrations that trace young stellar regions are seen at large radii in some nearby spiral galaxies (e.g. Gil de Paz et al. 2008; Zaritsky \& Christlein 2007). While the density of interstellar material at large radii tends to be too low to trigger large-scale star formation, localized density enhancements may occur as a result of compression from spiral density waves (e.g. Bush et al. 2008). The presence of a dark baryonic component in the disk plane could also enable star formation in areas where the gas density may otherwise appear to be too low (Revaz et al. 2009). Some fraction of the stars in the outer disk are probably migrants from smaller radii, as recent studies have shown that secular processes (e.g. Roskar et al. 2008) and radial mixing induced by interactions (e.g. Quillen et al. 2009) can contribute significantly to the stellar contents of the outer regions of disks.

The distribution of stars in the nearest spiral galaxies provide clues into the processes that populate the outermost regions of disks. Star-forming activity at large radii produces distinct signatures in galactic light and color profiles (e.g. Sanchez-Blazquez et al. 2009). As for secular effects, the processes that re-distribute stars throughout disks act in a cumulative manner on stellar orbits, with the result that stars that have moved the furthest from their places of birth will tend to be the oldest - stars with progressively older ages may thus be found at progressively larger galactocentric distances (e.g. Roskar et al. 2008), in contradiction to what might niavely be expected due to inside-out disk formation.

The present letter is part of a larger study of young and intermediate age stars throughout the disk of M33 (Davidge et al. 2011, in preparation). The entire dataset consists of five MegaCam pointings, and here we examine the distribution of stars in an area that includes two of the most remote star-forming complexes in M33. A distance modulus of 24.93 (Bo- 
nanos et al. 2006) is adopted. Recent distance modulus estimates for M33 show a spread of a few tenths of a dex, and the Bonanos et al. value was selected because it is based on eclipsing binaries, which are a primary distance indicator.

\section{OBSERVATIONS, REDUCTIONS, \& PHOTOMETRIC MEASUREMENTS}

The data were recorded as part of the 2009B MegaCam (Boulade et al. 2003) observing queue on the Canada-France-Hawaii Telescope (CFHT). The MegaCam detector is a mosaic of $362048 \times 4612 \mathrm{E} 2 \mathrm{~V}$ CCDs that are deployed in a $4 \times 9$ format. A $0.96 \times 0.94$ degree $^{2}$ area is imaged with 0.18 arcsec pixel $^{-1}$ sampling. Five $150 \mathrm{sec}$ exposures were recorded in $g^{\prime}$, and ten $440 \mathrm{sec}$ exposures were obtained in $u^{\prime}$.

The initial processing of the data, which included bias subtraction and flat-fielding, was done with the ELIXER pipeline at the CFHT. The ELIXER-processed images were aligned, stacked, and then trimmed to the area of common exposure time. This paper deals with objects in a single $18 \times 13.5 \operatorname{arcmin}^{2}$ field that is centered at 01:34:43 Right Ascension and 31:22:00 Declination (E2000), and samples the disk of M33 at a galactocentric distance of $8.3 \mathrm{kpc}$, or 4 disk scale lengths. The southern half of this area, which includes the northern spiral arm and two young stellar complexes, is shown in Figure 1. Stars in the final images have FWHM $\sim 0.9$ arcsec.

The brightnesses of individual stars were measured with the PSF-fitting routine ALLSTAR (Stetson \& Harris 1988). The photometric calibration was defined using zeropoints and transformation coefficients computed from standard star observations that were recorded during 2009B. Sources that depart from the trend between magnitude and the photometric error computed by ALLSTAR, which tend to be non-stellar in appearance (e.g. Davidge 2010), were removed from the photometric catalogue.

\section{RESULTS}

The $\left(u^{\prime}, g^{\prime}-u^{\prime}\right)$ CMDs of stars in the 'Spiral Arm' and 'Outer Disk' areas indicated in Figure 1 are shown in Figure 2. The 'Spiral Arm' region covers the diffuse distribution of stars in the northern spiral arm, while the 'Outer Disk' area covers the remainder of the field. There is a large number of bright main sequence stars in the Spiral Arm CMD, and a comparison with $\mathrm{Z}=0.004$ isochrones from Girardi et al. (2004) indicates that stars with ages from $\leq 10 \mathrm{Myr}$ to $\geq 100 \mathrm{Myr}$ are detected. This metallicity was selected based on the 
oxygen abundance seen throughout much of the M33 disk (e.g. Magrini et al. 2010), although the predicted locus of the upper main sequence is not sensitive to the adopted metallicity. The main sequence in the Outer Disk CMD is much less pronounced than in the Spiral Arm CMD; still, that modest numbers of young and intermediate age main sequence stars are present indicates that a diffusely distributed, young stellar component occurs outside of the main body of the spiral arm.

The locations on the sky of stars in three areas of the $\left(u^{\prime}, g^{\prime}-u^{\prime}\right)$ CMD, marked in Figure 2, are shown in Figure 1. There are obvious age-related differences, in the sense that the stars in the 10 Myr sample tend to group together more than those in the 40 and 100 Myr samples. Even though the stellar distribution becomes more diffuse towards older ages, the northern spiral arm can still be identified in the $100 \mathrm{Myr}$ sample. While not shown here, the overall distribution of older samples (e.g. those with $u^{\prime}$ between 24.5 and 25.5 , and $u^{\prime}-g^{\prime}$ between 0 and 1 , which have an age $\sim 200 \mathrm{Myr}$ ), is even more diffuse.

The extent of clustering 2 can be quantified by examining the angular separations between star - star pairs, and we refer to the histogram distribution of all possible pairings as the star-star separation function ( $\mathrm{S} 3 \mathrm{~F})$. The S3F is based on a simple observable - an angular measurement on the sky - and yields information about the large scale distribution of objects that can be difficult to quantify by eye. The gaps between the CCDs do not significantly affect the S3Fs, as these amount to only $\sim 4 \%$ of the field covered.

We first consider the S3F of sources in the northern half of the field. This area is well offset from the disk, and contains a mix of halo stars and unresolved galaxies, but few - if any - young or intermediate age stars belonging to M33. Thus, this area serves as a control for investigating the distribution of objects at smaller radii. The S3F of objects in the northern half of the field that fall within the $100 \mathrm{Myr}$ region of the CMD is shown in the bottom panel of Figure 3. The gradual drop-off in the separation frequency at separations $>450--500$ arcsec is due to the finite size of the area sampled. The S3F is not symmetric because the field is not square. The inflexion point of the S3F of uniformly distributed objects will occur at a scale that is roughly one half the length of the shortest axis of the area examined, which is $425 \operatorname{arcsec}(2 \mathrm{kpc}$ ), and this matches approximately the inflexion point in the bottom panel of Figure 3.

The S3Fs of main sequence stars in the southern half of the MegaCam sub-panel are shown in the top three panels of Figure 3. These S3Fs clearly differ from the S3F of sources

\footnotetext{
${ }^{2}$ In this paper, clustering refers to the grouping of stars over a range of spatial scales. This includes, but is not restricted to, objects that are in 'star clusters', which typically subtend only a few parsecs, and so are not resolved with these data.
} 
in the northern half of the MegaCam data, due to stellar grouping in M33. The S3F of the 10 Myr sample contains substantial signal at separations $r<250 \operatorname{arcsec}(<1.1 \mathrm{kpc})$, and the width of the peak at small separations indicates that the youngest stars are grouped on scales $<150 \operatorname{arcsec}(<700 \mathrm{pc})$. This is comparable to the dimensions of star-forming complexes in nearby galaxies (e.g. Efremov 1995), as well as the scale of coherent star-formation in M33 (Sanchez et al. 2010). There is a second peak in the 10 Myr S3F near $r \sim 450(2.1$ $\mathrm{kpc}$ ) arcsec, and this occurs because the two young stellar concentrations in this field beat against each other in the separation measurements; the separation between the two clumps in the upper right hand panel agrees with that between the two most prominent clumps in Figure 1.

When compared with the 10 Myr S3F, clustering signatures are broader and have a smaller amplitude in the S3F of the $40 \mathrm{Myr}$ sample. The majority of stars in the 40 Myr sample are separated by distances up to at least $300 \operatorname{arcsec}(1.4 \mathrm{kpc})$. Even at this comparatively young age, stars have moved distances that are large enough to significantly blur clustering signatures in the S3F.

There is a more-or-less uniform signal in the 100 Myr S3F between 150 and 400 arcsec (0.7 and $1.9 \mathrm{kpc})$, with no evidence of clustering at separations $<150 \operatorname{arcsec}(<0.7 \mathrm{kpc})-$ large stellar complexes evidently dissipate over $\sim 100$ Myr timescales in this part of M33. In fact, the width of the ramp-up in the 100 Myr S3F suggests that the minumum star-star separation is typically $\sim 50$ arcsec, or $\sim 0.25 \mathrm{kpc}$, for stars of this age, while the onset of the plateau in the S3F suggests that the typical star-star separation is at least 150 arcsec, or $\sim 0.7 \mathrm{kpc}$.

\section{DISCUSSION \& SUMMARY}

The star-star separation function (S3F) has been used to investigate the projected distribution of main sequence stars in the northern disk of M33. Two young stellar complexes produce significant signal in the S3F of stars with ages $\sim 10 \mathrm{Myr}$ at separations $r<150$ $\operatorname{arcsec}(d<0.7 \mathrm{kpc})$. However, signatures of clustering are greatly diminished among stars

with ages $\sim 40 \mathrm{Myr}$, and the smooth S3F of stars with ages $\sim 100 \mathrm{Myr}$ suggests that there is little if any large-scale clustering among these stars. Thus, large scale stellar structures in this part of M33 evidently dissipate over time scales $\leq 100 \mathrm{Myr}$.

Stellar complexes in the outer regions of disks may be subjected to disruption mechanisms that differ from those in the main body of the disk. There is evidence for heating by halo structures in the outer regions of nearby galaxies (e.g. Martin \& Kennicutt 2001), and 
dynamical measurements suggest that halo bombardment becomes a significant source of heating at 4 disk scale lengths in nearby spirals (Herrmann et al. 2009), and this is the part of the M33 disk that we examine here. The broad, evenly distributed signal in the $100 \mathrm{Myr}$ S3F between 150 and $450 \operatorname{arcsec}(0.7-2.1 \mathrm{kpc})$ results from random motions on the order of $\sim 20 \mathrm{~km} \mathrm{sec}^{-1}$, and this is comparable to the outer disk extraplanar motions measured by Herrmann et al. (2009). Putman et al. (2009) find that HI in the outer regions of M33 has a velocity dispersion of $18.5 \mathrm{~km} \mathrm{sec}^{-1}$, and suggest that this may be a relic of an interaction within the past few Gyr between M31 and M33.

Newly formed stellar systems will be more prone to disruption if they have a low star formation efficiency (SFE), as feedback will remove gas early-on, thereby reducing - perhaps catastrophically - the gravitational field of the nascent system (Lada \& Lada 2003). A general trend for the SFE to diminish towards larger radii is seen in nearby galaxies (Leroy et al. 2008). This result is based on measurements made over kpc spatial scales, which is comparable to the sizes of the large structures probed here,

Star clusters are sub-structures within the large-scale complexes that are investigated here. The largest disk star clusters in M33 subtend $\leq 2 \operatorname{arcsec}$ (San Roman et al. 2010), and so fall in the smallest bin in the S3F. The signal in the S3F of the 100 Myr sample in the 0 - 20 arcsec bin is markedly smaller than in the 10 Myr sample, and if this trend extends to sub-arcsec sizes then this will be consistent with stellar clusters dissipating over $\sim 0.1$ Gyr timescales. In fact, the spatial distribution of star clusters with ages $<0.1-0.3$ Gyr in M33 is more compact than that of stars with the same age (Sarajedini \& Mancone 2007; Roman et al. 2010), suggesting that young star clusters in M33 dissipate over time spans that are less than a few tenths of a Gyr.

The disruption timescale of star clusters depends on a number of factors, including the rate at which remnant gas is removed from the cluster, the local environment, and twobody relaxation (e.g. summary by Elmegreen \& Hunter 2010). Gratier (2010) find that the masses of molecular clouds decrease with increasing radius in M33, and this should result in lower star cluster masses, which in turn may lead to a comparatively rapid disruption timescale for clusters in the outer regions of M33. Lamers et al. (2005a) estimates that a $10^{4}$ $\mathrm{M}_{\odot}$ cluster in the main body of M33 typically disrupts after $\sim 1$ Gyr. Assuming no radial changes in the sources of dynamical heating, the ambient mass mixture that dominates the gravitational field, and the mean SFE within M33, then if the cluster disruption timescale $\propto$ mass $^{\gamma}$, where $\gamma=0.62$ (Baumgardt \& Makino 2003; Lamers et al. 2005b), then the majority of stars in the outer disk of M33 formed in clusters with masses $\leq 50-250 \mathrm{M}_{\odot}$ if they are disrupted on timescales of $\sim 100 \mathrm{Myr}$. This characteristic cluster mass is roughly two orders of magnitude lower than the peak of the solar neighborhood initial cluster mass 
function predicted by Parmentier et al. (2008) and Kroupa \& Boily (2002). In fact, this is an upper limit to the initial cluster mass, in the sense that the pace with which clusters dissolve depends on factors such as the local mass density and the initial cluster mass, and a $10^{4} \mathrm{M}_{\odot}$ cluster in the peripheral regions of the M33 disk would be even longer lived than predicted by Lamers et al. (2005a). Thus, if star clusters are disrupted over $\sim 0.1 \mathrm{Gyr}$ timescales in the outer regions of M33 then we predict that the star clusters found there will have (1) young ages, and (2) lower masses than those at smaller radii.

We close by noting that the orderly large scale motions induced by secular processes are probably not significant among stars of the age considered here, given that the rotation period of the M33 disk is $200-300$ Myr (Corbelli \& Salucci 2000). Rather, stars with ages $\sim 100 \mathrm{Myr}$ in this part of M33 appear to have obtained random stellar motions that allow them to populate regions up to $\sim 2 \mathrm{kpc}$ from where they formed. This effectively pushes out the observational boundary of the young disk.

\section{REFERENCES}

Baumgardt, H., \& Makino, J. 2003, MNRAS, 340, 227

Bonanos, A. Z., et al. 2006, ApJ, 652, 313

Boulade, O., et al. 2003, Proc. SPIE, 4841, 72

Bush, S. J., Cox, C. J., Hernquist, L., Thilker, D., \& Younger, J. D. 2008, ApJ, 683, L13

Corbelli, E., \& Salucci, P. 2000, MNRAS, 311, 441

Davidge, T. J. 2006, ApJ, 641, 822

Davidge, T. J. 2010, ApJ, 725, 1342

Dong, H., Calzetti, D., Regan, M., Thilker, D., Bianchi, L., Meurer, G. R., \& Walter, F. 2008, AJ, 136, 479

Efremov, Y. N. 1995, AJ, 110, 2757

Elmegreen, B. G., \& Hunter, D. A. 2010, ApJ, 712, 604

Gil de Paz, A., et al. 2008, Formation and Evolution of Galaxy Disks, ASP Conf. Vol. 396, ed J. G. Funes \& E. M. Corsini, pp. 197

Girardi, L., Grebel, E. K., Odenkirchen, M., \& Chiosi, C. 2004, A\&A, 422, 205

Gratier, P. 2010, PhD thesis, Universite Bordeaux 1, in preparation

Herrmann, K. A., Ciardullo, R., \& Sigurdsson, S. 2009, ApJ, 693, L19

Kroupa, P., \& Boily, C. M. 2002, MNRAS, 336, 1188 
Lada, C. J., \& Lada, E. A. 2003, ARA\&A, 41, 57

Lamers, H. J. G. L. M., Gieles, M., \& Portegies Zwart, S. F. 2005a, A\&A, 429, 173

Lamers, H. J. G. L. M., Gieles, M., Bastian, N., Baumgardt, H., Kharchenko, N. V., \& Portigies Zwart, S. 2005b, A\&A, 441, 117

Leroy, A. K., Walter, F., Brinks, E., Bigiel, F., de Blok, W. J. G., Madore, B., \& Thornley, M. D. 2008, AJ, 136, 2782

Magrini, L., Stanghellini, L., Corbelli, E., Galli, D., \& Villaver, E. 2010, A\&A, 512, 63

Martin, C. L., \& Kennicutt, R. C. Jr. 2001, ApJ, 555, 301

Parmentier, G., Goodwin, S. P., Kroupa, P., \& Baumgardt, H. 2008, ApJ, 678, 347

Pierce, M. J., \& Tully, R. B. 1992, ApJ, 387, 47

Pohlen, M., \& Trujillo, I. 2006, A\&A, 454, 759

Putnam, M. E., et al. 2009, ApJ, 703, 1486

Quillen, A. C., Minchev, I., Bland-Hawthorn, J., \& Haywood, M. 2009, MNRAS, 397, 1599

Revaz, Y., Pfenniger, D., Combes, F., \& Bournaud, F. 2009, A\&A, 501, 171

San Roman, I., Sarajedini, A., \& Aparicio, A. 2010, ApJ, 720, 1674

Sarajedini, A., \& Mancone, C. L. 2007, AJ, 134, 447

Roskar, R., Debattista, V. P., Quinn, T. R., Stinson, G. S., \& Wadsley, J. 2008, ApJ, 684, L79

Sanchez, N., Anez, N., Alfaro, E. J., \& Odekon, M. C. 2010, ApJ, 720, 541

Sanchez-Blazquez, P., Courty, S., Gibson, B. K., \& Brook, C. B. 2009, MNRAS, 398, 591

Schlegel, D. J., Finkbeiner, D. P., \& Davis, M. 1998, ApJ, 500, 525

Stetson, P. B., \& Harris, W. E. 1988, AJ, 96, 909

Zaritsky, D., \& Christlein, D. 2007, AJ, 134, 135 


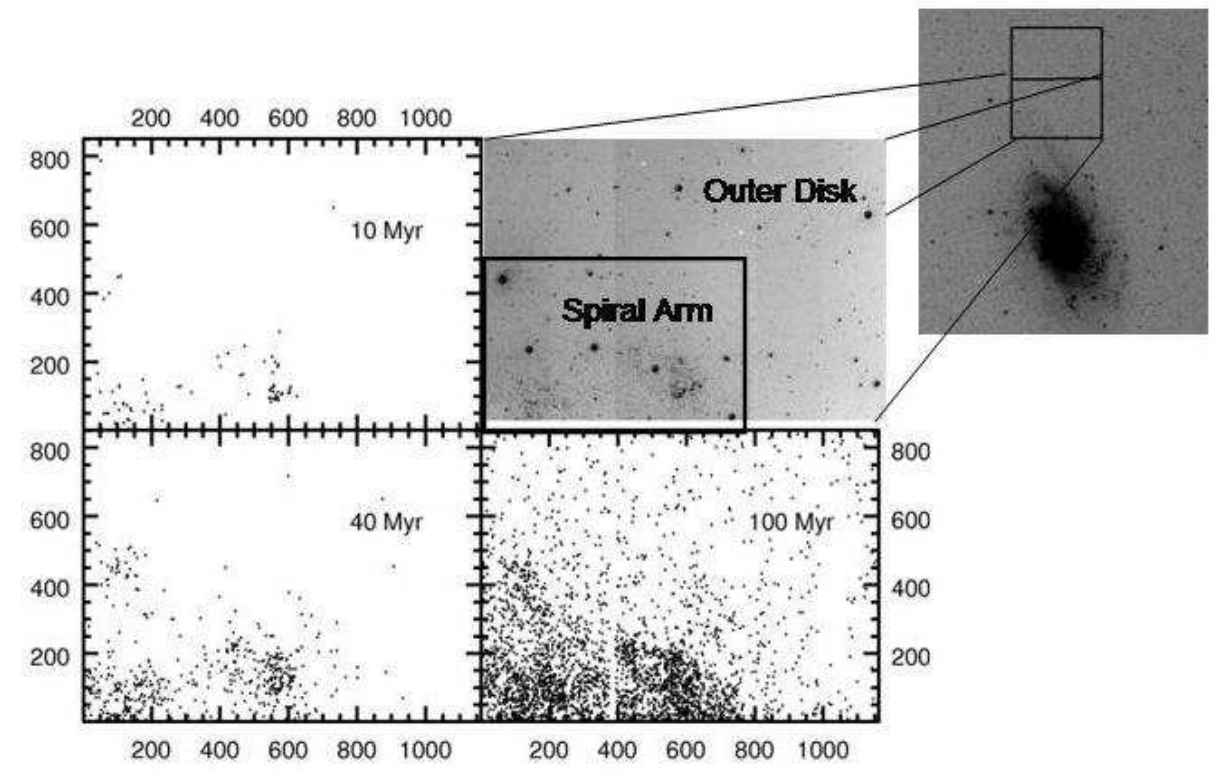

Fig. 1. - The distribution of objects in the area studied. North is at the top, and East is to the left. The field location is indicated on a $50 \times 60 \mathrm{arcmin}^{2}$ section of the blue DSS; the line in this inset divides the field into the northern and southern halfs that are discussed in $\S 3$. A $18 \times 13.5$ arcmin $^{2}$ area is covered in each of the remaining panels, with the axes indicating distances in arcsec as measured from the south east corner. The areas containing the stars that are used to construct the CMDs in Figure 2 are indicated on the $u^{\prime}$ image. The locations of objects in three photometrically-selected samples are shown in the other panels. Note that (1) density enhancements due to two large young stellar complexes are seen in the 10 and 40 Myr samples, and (2) significant numbers of stars with ages $\sim 100$ Myr are seen to the north west of the main body of the spiral arm. 


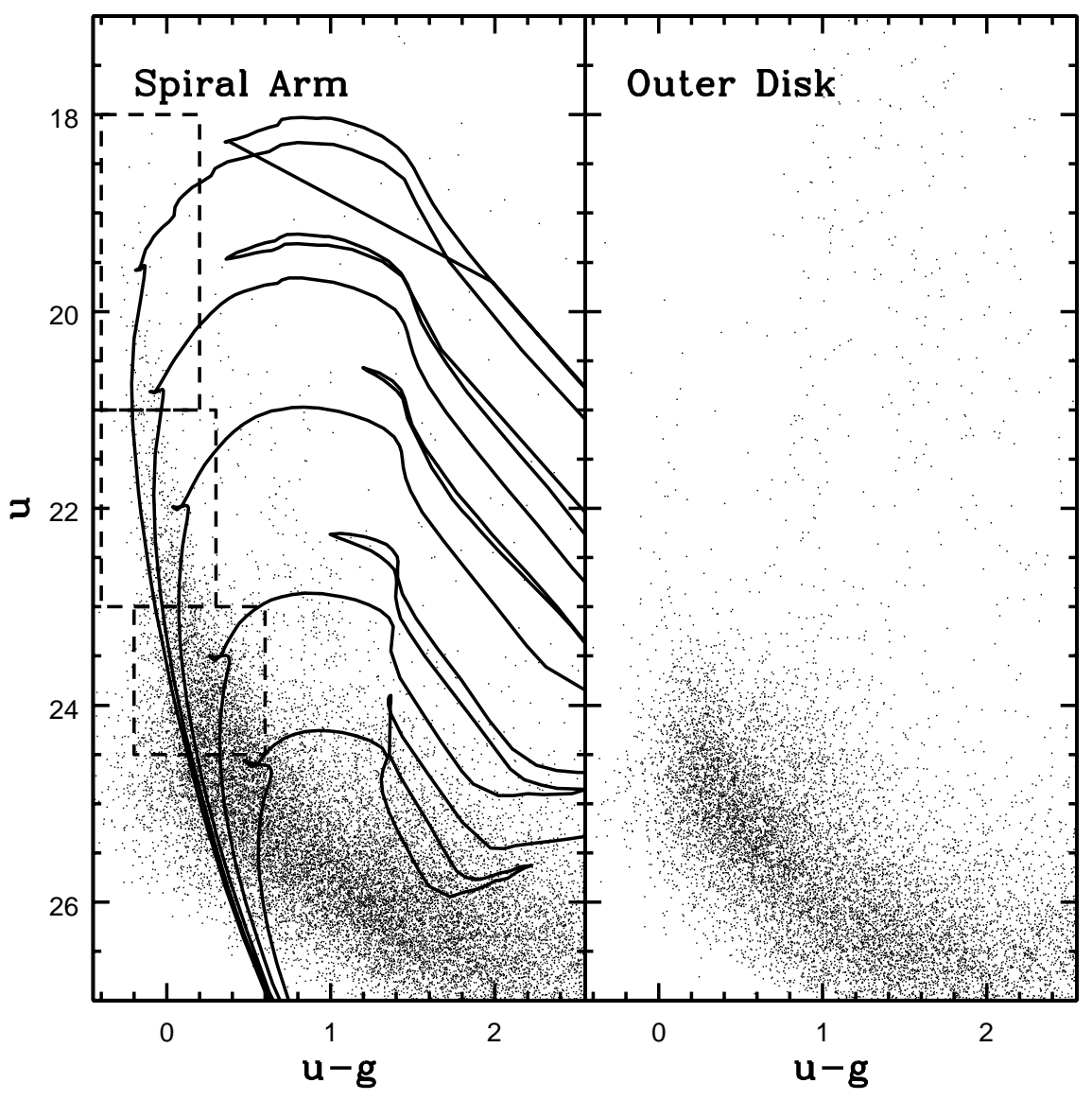

Fig. 2.- The $\left(u^{\prime}, u^{\prime}-g^{\prime}\right)$ CMDs of sources in the 'Spiral Arm' and 'Outer Disk' regions indicated in Figure 1. The solid lines are $\mathrm{Z}=0.004$ isochrones from Girardi et al. (2004) with ages $10 \mathrm{Myr}, 20 \mathrm{Myr}, 40 \mathrm{Myr}, 100 \mathrm{Myr}$, and $200 \mathrm{Myr}$. A foreground extinction $\mathrm{A}_{B}=0.181$ (Schlegel et al 1998) and an internal extinction $A_{B}=0.16$ (Pierce \& Tully 1992) have been adopted. The dashed lines mark the areas on the CMDs from which stars are extracted to investigate clustering. MSTO stars near the centers of these boxes have ages $10 \mathrm{Myr}, 40$ Myr, and 100 Myr. 


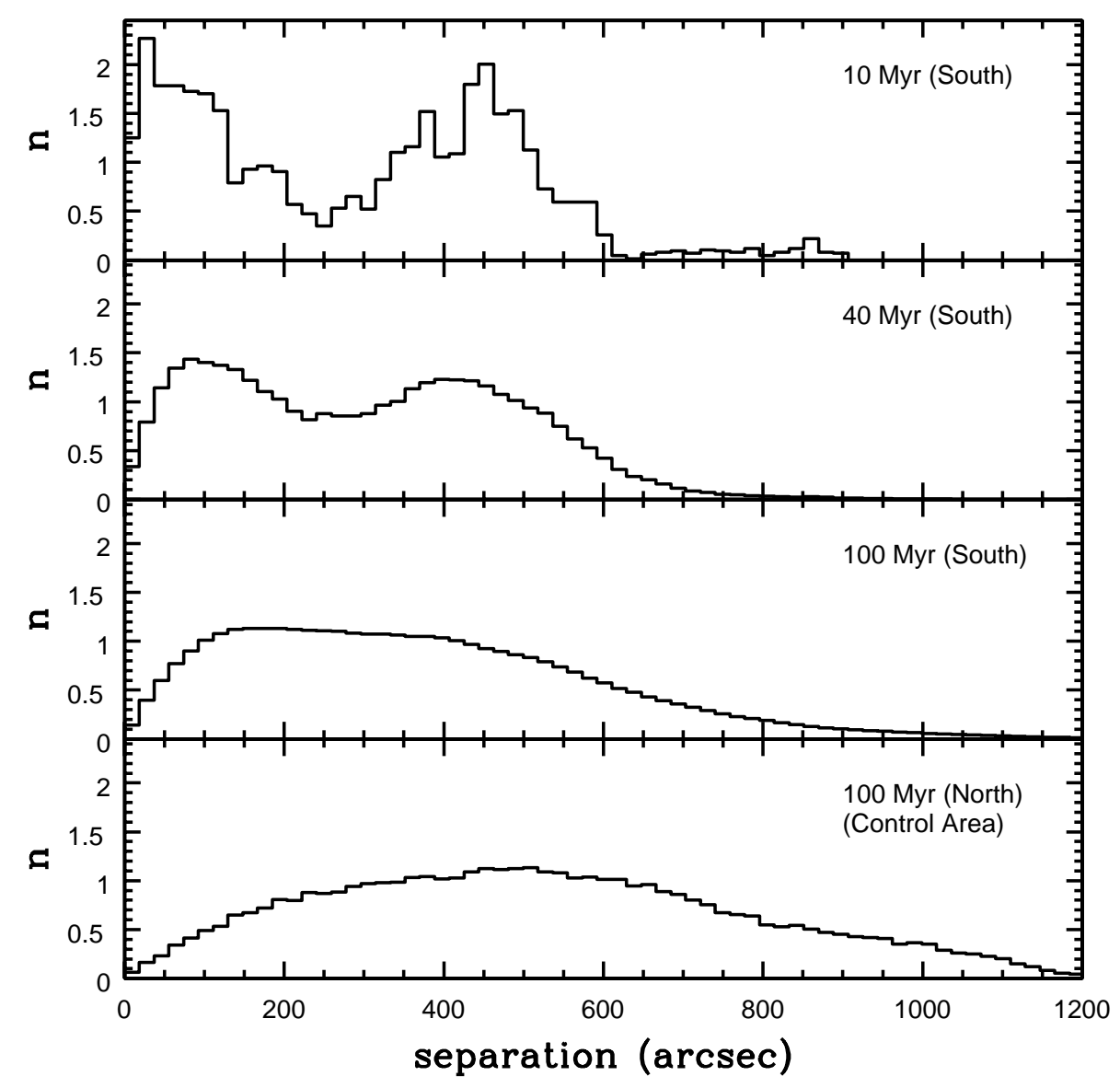

Fig. 3.- The star-star separation functions (S3Fs) of sources in the northern and southern half of the MegaCam data. $20 \operatorname{arcsec}(\sim 90$ pc) binning has been used, and ' $\mathrm{n}$ ' is the number of sources in each bin normalized to the mean signal between 200 - 550 arcsec, which is the range of separations that dominate disk stars in this field. The spatial scales investigated here probe large-scale stellar complexes, rather than classical star clusters, the majority of which in M33 tend to have angular sizes that are comparable to the seeing disk. The 100 Myr S3F of the northern half of the field is that of a more-or-less uniformly distributed ensemble of objects. In contrast, the S3Fs of sources in the southern half of the field show enhanced signal at separations $\leq 400$ arcsec. The prominent peak in the $10 \mathrm{Myr}$ S3F at low separations indicates that the youngest stars group over angular scales $\leq 150$ arsec, or projected distances of roughly $700 \mathrm{pc}$; the second peak is due to sources in the two complexes beating against each other. Aside from the broad signature produced by the main body of the northern spiral arm, there is no evidence of structures in the 100 Myr S3F, indicating that the processes that dissolve large scale star-forming regions in this part of M33 do so on times scales $\leq 100$ Myr. 